\title{
Clock Skew Compensation Algorithm Immune to Floating-Point Precision Loss
}

\author{
Kyeong Soo Kim, Senior Member, IEEE, and Seungyeop Kang
}

\begin{abstract}
We propose a novel clock skew compensation algorithm based on Bresenham's line drawing algorithm. The proposed algorithm can avoid the effect of limited floating-point precision (e.g., 32-bit single precision) on clock skew compensation and thereby provide high-precision time synchronization even with resource-constrained sensor nodes in wireless sensor networks.
\end{abstract}

Index Terms-Clock skew compensation, Bresenham's algorithm, time synchronization, floating-point arithmetic, wireless sensor networks.

\section{INTRODUCTION}

C LOCK skew compensation is an essential component of time synchronization in wireless sensor networks (WSNs), which provides a common time frame among network nodes [1]. Because typical clock skew compensation algorithms are involved with floating-point arithmetic, their performance on the platforms with lower computational resources-e.g., 32-bit single-precision floating-point format on resource-constrained WSN sensor nodes-is not up to the predicted performance from theories or simulation experiments [2].

To address the issue of the limited precision floating-point arithmetic in clock skew compensation, therefore, we propose a novel scheme based on Bresenham's line drawing algorithm [3] immune to floating-point precision loss.

\section{Clock Models}

Without loss of generality, we confine our discussions to a network with one head node and one sensor node in this letter, where we describe the hardware clock $T$ of the sensor node with respect to the reference clock $t$ of the head node using the first-order affine clock model [4]:

$$
T(t)=(1+\epsilon) t+\theta,
$$

where $\epsilon \in \mathbb{R}$ and $\theta \in \mathbb{R}$ denote the clock skew and offset, respectively; $(1+\epsilon) \in \mathbb{R}_{+}$in $(1)$ is also called clock frequency ratio in the literature. Because we focus on the clock skew compensation, we simplify (1) by setting $\theta$ to 0 as follows:

$$
T(t)=(1+\epsilon) t
$$

Because our proposal in this paper compensates for the clock skew only (equivalently clock frequency ratio), it is different

This work was supported in part by the Postgraduate Research Scholarships (under Grant PGRS1912001) and the Key Programme Special Fund (under Grant KSF-E-25) of Xi'an Jiaotong-Liverpool University.

K. S. Kim is with the Department of Communications and Networking, School of Advanced Technology, Xi'an Jiaotong-Liverpool University, Suzhou 215123, P. R. China (e-mail: Kyeongsoo.Kim@xjtlu.edu.cn).

S. Kang is with the Department of Mechatronics and Robotics, School of Advanced Technology, Xi'an Jiaotong-Liverpool University, Suzhou 215123 P. R. China (e-mail: S.Kang18@student.xjtlu.edu.cn). from ratio-based schemes like ratio-based time synchronization protocol (RSP) [5] compensating both clock skew and clock offset. Note that the time synchronization schemes based on the reverse two-way message exchange proposed in [6] are based on the offset-free clock model of (2), where the clock offset is independently compensated for at the head node, while the sensor node only synchronizes the frequency of its logical clock to that of the reference clock.

Compensating for the clock skew from the hardware clock $T$ in (2), we can obtain the logical clock $\hat{t}$ of the sensor nodei.e., the estimation of the reference clock $t$ given the hardware clock $T$-as follows: For $t_{i}<t \leq t_{i+1}(i=0,1, \ldots)$,

$$
\hat{t}(T(t))=\hat{t}\left(T\left(t_{i}\right)\right)+\frac{T(t)-T\left(t_{i}\right)}{1+\hat{\epsilon}_{i}},
$$

where $t_{i}$ is the reference time for the $i$ th synchronization and $\hat{\epsilon}_{i}$ is the estimated clock skew from the $i$ th synchronization 1

The impact of the limited precision in floating-point arithmetic on clock skew compensation is investigated in [2]: The major finding is that the division of floating-point numbers in (3) incurs substantial precision loss at typical WSN platforms with 32-bit single precision. In case of the time synchronization scheme based on the reverse two-way message exchange [6], because the logical clock updates at sensor nodes in (3) requires accurate floating-point division and has a recursive nature, the impact of the precision loss on the logical clock is accumulated over time.

\section{Clock Skew COMPENSATION BASED ON BRESENHAM'S ALGORITHM}

The major issue in the clock skew compensation based on (3) is the floating-point division required for the calculation of the second term $\frac{T(t)-T\left(t_{i}\right)}{1+\hat{\epsilon}_{i}}$, i.e., the skew-compensated increment of the hardware clock since the $i$ th synchronization. In this section, we describe how to obtain the second term of (3) using only integer addition/subtraction and comparison based on the Bresenham's algorithm.

Note that, though the modeling of clocks in Section III is based on the continuous-time affine clock model, clocks in digital communication systems are basically discrete counters [7]; timestamp values exchanged between a head and sensor nodes or recorded for events and measurements at sensor nodes are based on the readings of discrete counters.

Let $\frac{D}{A}$ be the inverse of a clock frequency ratio (i.e., $\frac{1}{1+\epsilon_{i}}$ ) estimated based on two positive integers $D$ and $A ; D$ and $A$ represent interdeparture and interarrival times of packets or

\footnotetext{
${ }^{1}$ The sensor node does not know the reference clock $t$ as such but only the hardware clock corresponding to $t$-i.e., $T(t)$-during the operation.
} 


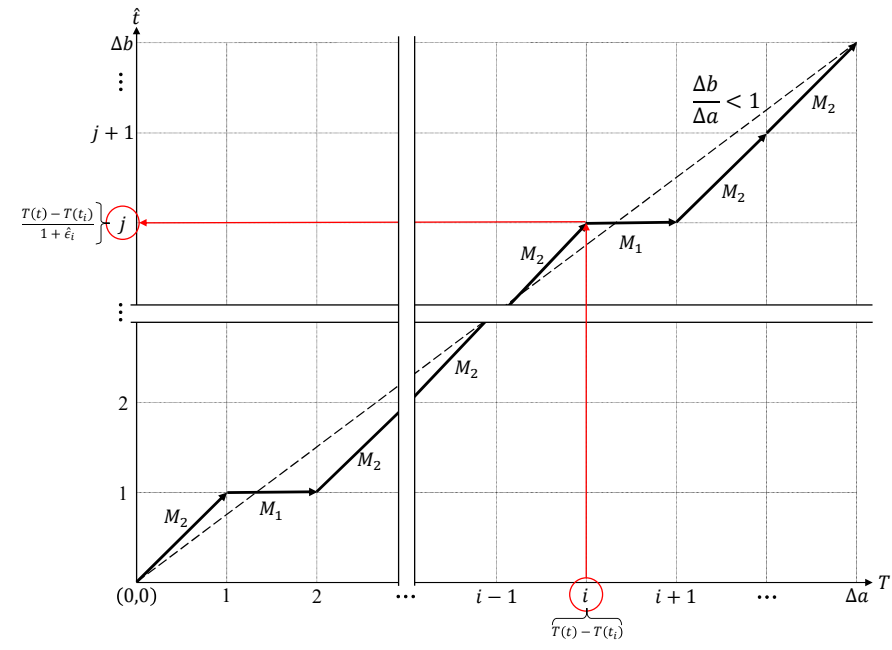

Fig. 1. Clock skew compensation based on Bresenham's line drawing algorithm [3] for the case of $\frac{\Delta b}{\Delta a}<1$.

their cumulative sums from the previous synchronization $\left.[8]\right|^{2}$ Based on the Bresenham's algorithm [3], we can obtain the skew-compensated increment of the hardware clock as follows: For $\frac{D}{A}<1$, we set $\Delta a$ and $\Delta b$ to $A$ and $D$, respectively, and calculate $\nabla_{i}$ using the following recursive relation ${ }^{3}$

$$
\begin{aligned}
\nabla_{0} & =2 \Delta b-\Delta a, \\
\nabla_{i+1} & = \begin{cases}\nabla_{i}+2 \Delta b-2 \Delta a & \text { if } \nabla_{i} \geq 0, \\
\nabla_{i}+2 \Delta b & \text { otherwise. }\end{cases}
\end{aligned}
$$

Then, from the origin and on, we determine each movement based on $\nabla_{i}$ :

$$
\begin{cases}M_{1} & \text { if } \nabla_{i}<0, \\ M_{2} & \text { otherwise }\end{cases}
$$

where $M_{1}$ is a horizontal movement and $M_{2}$ is a diagonal movement shown in Fig. $11^{4}$ Lemma 1 shows that $\nabla_{i}$ is bounded.

Lemma 1. $\nabla_{i}$ satisfies the following inequality:

$$
\left|\nabla_{i}\right|<2 \Delta a \text {. }
$$

Proof: See Appendix A

Unlike the line drawing, the skew compensation in our case does not need all the intermediate points between the origin and the point under consideration; we need only the $y$ coordinate of a point given its $x$ coordinate (e.g., $j$ given $i$ in Fig. 11, where $x$ coordinate is the increment of the hardware clock and $y$ coordinate is its skew-compensated increment for the logical clock $\hat{t}(T)$. Therefore, we cannot use Bresenham's original algorithm as it is, especially when $D$ and $A$ are large and there are sparse events/measurements.

\footnotetext{
${ }^{2}$ In the following, we do not consider the effect of the random noise components during the estimation of the clock frequency ratio in the values of $D$ and $A$ to clearly assess the improvement made by the proposed algorithm over conventional clock skew compensation based on floating-point division.

${ }^{3}$ The case of $\frac{D}{A}>1$ will be handled in Theorem 1 and the index starts from 0 to make mapping easier between $\nabla_{i}$ and the $x$ coordinate of a point (e.g., $\nabla_{0}$ is for $(0,0)$ ).

${ }^{4}$ The coordinates are shifted by a pair of the initial values of hardware and logical clocks, e.g., $\left(T\left(t_{i}\right), \hat{t}\left(T\left(t_{i}\right)\right)\right)$ in 3 .
}

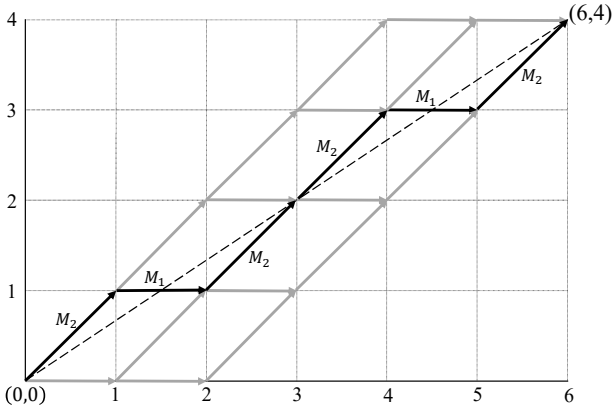

Fig. 2. Example of all possible paths from the origin to a given destination point based on $M_{1}$ and $M_{2}$ movements.

To extend Bresenham's algorithm for skew compensation, let's consider the example shown in Fig. 2, where the only valid path from $(0,0)$ to $(6,4)$ according to Bresenham's algorithm is indicated by black arrows while alternative paths are by gray arrows. Though the alternative paths are not useful for line drawing, they can reach the same destination and thereby provide the correct $y$ coordinate. In fact, Lemma 1 shows that $\nabla_{i}$ in Bresenham's algorithm depends only on the number of $M_{2}$, not the exact sequence of $M_{1}$ and $M_{2}$, during the total $i$ movements. Therefore, we extend $\nabla_{i}$ of Bresenham's algorithm to the points on all possible paths from the origin to a given destination of $(\Delta a, \Delta b)$ based on the two movements of $M_{1}$ and $M_{2}$ and define $\bar{\nabla}_{i}(j)$ as follows:

Definition 1. Given a destination point $(\Delta a, \Delta b)$, we define valid point set $\mathscr{V}(\Delta a, \Delta b)$ as a set of the points on a valid path from $(0,0)$ to $(\Delta a, \Delta b)$ according to Bresenham's algorithm.

Definition 2. Given a point $(i, j) \in \mathscr{V}(\Delta a, \Delta b)$, we define backward-reachable set $\mathscr{B}(i, j)$ as a set of the points that can reach $(i, j)$ by any combination of the movements $M_{1}$ and $M_{2}$ as follows:

$$
\begin{aligned}
& \mathscr{B}(i, j) \triangleq \\
& \{(k, l) \mid 0 \leq k<i, \max (0, k-\Delta a+\Delta b) \leq l \leq \min (k, j)\} .
\end{aligned}
$$

Definition 3. For a point $(i, j) \in \mathscr{B}(\Delta a, \Delta b)$, we define $\bar{\nabla}_{i}(j)$ as follows:

$$
\bar{\nabla}_{i}(j) \triangleq 2(i \Delta b-j \Delta a) .
$$

Note that $\bar{\nabla}_{i}(j)=\nabla_{i}, \forall(i, j) \in \mathscr{V}(\Delta a, \Delta b)$. Lemma 2 shows the property of $\bar{\nabla}_{i}(j)$ essential to the extension of Bresenham's algorithm to clock skew compensation:

Lemma 2. A point $(i, j) \in \mathscr{V}(\Delta a, \Delta b)$ is reachable from any point $(k, l) \in \mathscr{B}(i, j)$ if we apply (4)-(6) using $\bar{\nabla} .(\cdot)$ instead of $\nabla$..

Proof: See Appendix B

Now we can prove the main theorem for the clock skew compensation based on the extension of Bresenham's algorithm, which can eliminate the effect of precision loss on floating-point arithmetic:

Theorem 1. Given the hardware clock $i$, we can obtain its skew-compensated clock $j$ as follows: 


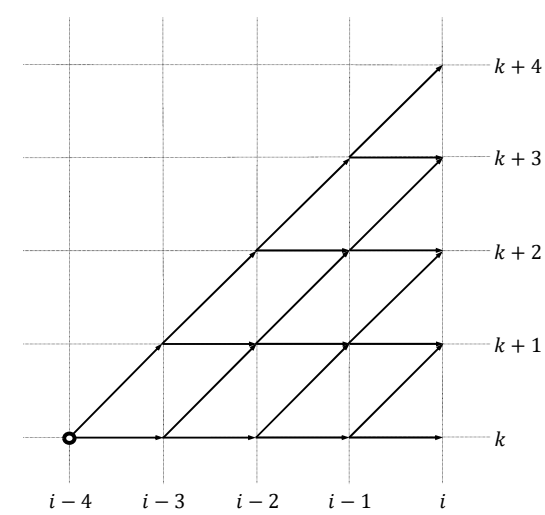

Fig. 3. Example of common starting points reaching all possible candidate points for the case of $\frac{\Delta b}{\Delta a}<1$.

Case 1. $\frac{D}{A}<1$ : The skew-compensated clock $j$ satisfies

$$
i \frac{D}{A}-1<j<i \frac{D}{A}+1 \text {. }
$$

Unless $i \frac{D}{A}$ is an integer, there are two values satisfying 107 . Due to the effect of limited floating-point precision, however, we cannot know the exact value of $i \frac{D}{A}$. In this regard, (10) can be extended to include the effect of the precision loss:

$$
i \frac{D}{A}-1-\varepsilon<j<i \frac{D}{A}+1+\varepsilon,
$$

where $\varepsilon(>0)$ is the error due to the precision loss. Let $k, \ldots, k+l$ be the candidate values of $j$ satisfying (11). We determine $j$ by starting from the point $(i-l, k)$ and applying Bresenham's algorithm with $\bar{\nabla}_{i-l}(k)$ and on; $j$ is determined by the $y$ coordinate of the valid point whose $x$ coordinate is $i$.

Case 2. $\frac{D}{A}>1$ : In this case, we can decompose the skewcompensated clock $j$ into two components as follows:

$$
j=i \frac{D}{A}=i+i \frac{D-A}{A} .
$$

Now that $\frac{D-A}{A}<1$, we can apply the same procedure of Case 1 to the second component in (12) by setting $\Delta a$ and $\Delta b$ to $A$ and $D-A$, respectively. Let $\bar{j}$ be the result from the procedure. The skew-compensated clock $j$ is given by $i+\bar{j}$ as per (12).

Proof: See Appendix C

In summary, the clock skew compensation algorithm described in Theorem 1 allows us to bound the correct skewcompensated clock given a hardware clock by (11) and search for it from a nearby starting point-instead of the originusing the extension of Bresenham's algorithm based on $\bar{\nabla}$. (.). Fig. 3 illustrates an example of the starting point discussed in Theorem 1 .

Note that the case of $\frac{D}{A}>1$ is handled by setting $\Delta a$ and $\Delta b$ to $D$ and $A$ in Bresenham's original algorithm, where we need to find the skew-compensated clock $j$ on the $x$-axis given the hardware clock $i$ on the $y$-axis. The major issue is that there could be multiple points on the $x$-axis given a point in the $y$-axis due to the movement of $M_{1}$ (e.g., $(1,1)$ and $(2,1)$ in Fig. 11. The algorithm described in Theorem 1 can avoid such an issue resulting from the change of the axes by unifying both cases with the same procedure for $\frac{D}{A}<1$.
TABLE I

RESUlts of ClOCK SKew COMPENSATION.

\begin{tabular}{c|c|r|r|r|r|r}
\hline \multirow{2}{*}{ Algorithm } & \multirow{2}{*}{$i$} & \multicolumn{3}{|c|}{ Compensation error } & \multicolumn{2}{c}{ \# of iterations ${ }^{\dagger}$} \\
\cline { 3 - 7 } & & Min. & Max. & Avg. & Min. & Max. \\
\hline \multirow{3}{*}{ Single } & $1 \mathrm{e} 6$ & 0 & 0 & 0 & - & - \\
precision $^{\ddagger}$ & $1 \mathrm{e} 7$ & 0 & 0 & 0 & - & - \\
& $1 \mathrm{e} 8$ & -4 & 1 & -2.0004 & - & - \\
& $1 \mathrm{e} 9$ & -19 & 44 & $1.2382 \mathrm{e} 1$ & - & - \\
\hline \multirow{5}{*}{ Proposed } & $1 \mathrm{e} 6$ & -1 & 0 & $-4.9820 \mathrm{e}-1$ & 2 & 2 \\
& $1 \mathrm{e} 7$ & -1 & 0 & $-4.9685 \mathrm{e}-1$ & 3 & 4 \\
& $1 \mathrm{e} 8$ & -1 & 0 & $-5.0263 \mathrm{e}-1$ & 21 & 22 \\
& $1 \mathrm{e} 9$ & -1 & 0 & $-4.9708 \mathrm{e}-1$ & 201 & 202 \\
\hline
\end{tabular}

* With respect to $\left\lfloor i \frac{D}{A}\right\rfloor$ based on double precision.

$\dagger l$ in Theorem 1

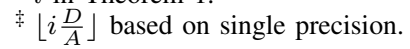

\section{A. Numerical Examples}

We apply the proposed algorithm to the representative cases whose results are summarized in Table I. We fix $D$ to $1,000,000$ and generate one million samples of $A$ whose clock skews are uniformly distributed in the range of $[-100 \mathrm{ppm}, 100 \mathrm{ppm}][9]$. The value of $\varepsilon$ is set to $10^{-7} i$ based on the analysis in [2, Section 2]; the minimum and the maximum values of $i$ (i.e., the hardware clock) in Table I correspond to $1 \mathrm{~s}$ and $1000 \mathrm{~s}$, respectively, at a sensor node running TinyOS whose synchronization limit is $1 \mu \mathrm{s}$ [10]. For the clock skew compensation by single and double-precision floating-point arithmetic, we round down the results to obtain integer values.

The results in Table I show that the clock skew compensation errors of the proposed algorithm with respect to the results based on double-precision floating-point arithmetic are bounded by $[-1,0]$, while those of the single-precision algorithm begins to increase when the hardware clock is $1 \mathrm{e} 8$. When we change rounding down to rounding off and rounding up for the results from double-precision and singleprecision algorithms, we still obtain similar results where the compensation errors of the proposed algorithm are always bounded by $[-1,1]$.

Note that $i=1 \mathrm{e} 9$ - corresponding to $1000 \mathrm{~s}$-is unlikely in a practical scenario because the maximum value of $i$ is limited by the synchronization interval, especially considering the cheap quartz crystal oscillator. Excluding the case of $i=1 \mathrm{e} 9$, therefore, we observe that the number of iterations for a typical value of $i$ is reasonable and that the computational complexity of the proposed algorithm is not a major issue. During the experiments, we also found that the upper and lower bounds in (11) based on $\varepsilon=10^{-7} i$ are very loose and that there is room for tighter bounds and thereby reducing the number of iterations, which would require further investigation on the tradeoff between computational complexity and precision loss in the proposed algorithm.

\section{CONClusions}

In this letter, we have proposed a novel clock skew compensation algorithm immune to floating-point precision loss, where we extend Bresenham's algorithm to the points on all 
possible paths from the origin to the destination based on the movements of $M_{1}$ and $M_{2}$ and unify the two cases of $\frac{D}{A}<1$ and $\frac{D}{A}>1$ with a common procedure. Through numerical examples, we have also demonstrated that the proposed algorithm can compensate for clock skew without being affected by precision loss unlike the existing schemes based on floatingpoint divisions.

\section{APPENDIX A}

PROOF OF LEMMA 1

Because $0<\frac{\Delta b}{\Delta a}<1$,

$$
\begin{aligned}
0 & <\Delta b<\Delta a, \\
0 & <2 \Delta b<2 \Delta a, \\
-\Delta a & <2 \Delta b-\Delta a<\Delta a, \\
\therefore-2 \Delta a & <\nabla_{0}<2 \Delta a .
\end{aligned}
$$

Let's assume $-2 \Delta a<\nabla_{i}<2 \Delta a$ for $i \geq 0$.

Case 1. $\nabla_{i} \geq 0$ :

$$
\begin{aligned}
0 & \leq \nabla_{i}<2 \Delta a, \\
2 \Delta b-2 \Delta a & \leq \nabla_{i}+2 \Delta b-2 \Delta a<2 \Delta b .
\end{aligned}
$$

From (13, we have $-2 \Delta a<2 \Delta b-2 \Delta a$ and $2 \Delta b<2 \Delta a$. So we obtain

$$
\begin{aligned}
-2 \Delta a & <\nabla_{i}+2 \Delta b-2 \Delta a<2 \Delta a, \\
\therefore-2 \Delta a & <\nabla_{i+1}<2 \Delta a .
\end{aligned}
$$

Case 2. $\nabla_{i}<0$ :

$$
\begin{aligned}
-2 \Delta a & <\nabla_{i}<0, \\
2 \Delta b-2 \Delta a & <\nabla_{i}+2 \Delta b<2 \Delta b .
\end{aligned}
$$

Again, from (13), we have $-2 \Delta a<2 \Delta b-2 \Delta a$ and $2 D<2 A$. So we obtain

$$
\begin{aligned}
& -2 \Delta a<\nabla_{i}+2 \Delta b<2 \Delta a, \\
\therefore- & 2 \Delta a<\nabla_{i+1}<2 \Delta a .
\end{aligned}
$$

(13)-17] completes the proof by mathematical induction.

\section{APPENDIX B}

\section{PROOF OF LEMMA 2}

We first show that $(i, j) \in \mathscr{V}(\Delta a, \Delta b)$ is reachable from $(i-1, l) \in \mathscr{B}(i, j)$, i.e., the points one step backward from it. Considering the movements of $M_{1}$ and $M_{2}$, we could have at most two such points, i.e., $(i-1, j-1)$ and $(i-1, j) 5^{5}$

Case 1. $(i-1, j-1) \in \mathscr{V}(\Delta a, \Delta b): \nabla_{i-1}$ should be equal to or greater than 0 so that we take $M_{2}$ and move to $(i, j)$. Now we have two subcases:

Case 1.1. Start from $(i-1, j-1):(i, j)$ can be reachable using $\bar{\nabla}_{i-1}(j-1)$ because $\bar{\nabla}_{i-1}(j-1)=\nabla_{i-1}$.

Case 1.2. Start from $(i-1, j): \bar{\nabla}_{i-1}(j)=\bar{\nabla}_{i-1}(j-1)-2 \Delta a$, so we have

$$
\begin{aligned}
&-2 \Delta a<\bar{\nabla}_{i-1}(j-1)<2 \Delta a\left(\because \bar{\nabla}_{i-1}(j-1)=\nabla_{i-1}\right), \\
&-4 \Delta a<\bar{\nabla}_{i-1}(j-1)-2 \Delta a<0, \\
&-4 \Delta a<\bar{\nabla}_{i-1}(j)<0 .
\end{aligned}
$$

\footnotetext{
${ }^{5}$ It is possible that $(i-1, j) \notin \mathscr{B}$ (e.g., $(1,1)$ in Fig. 2).
}

Because $\bar{\nabla}_{i-1}(j)<0$, we take $M_{1}$ and move to $(i, j)$.

Case 2. $(i-1, j) \in \mathscr{V}(\Delta a, \Delta b): \nabla_{i-1}$ should be less than 0 so that we take $M_{1}$ and move to $(i, j)$. Again, we have two subcases:

Case 2.1. Start from $(i-1, j-1): \bar{\nabla}_{i-1}(j-1)=\bar{\nabla}_{i-1}(j)+2 \Delta a$, so we have

$$
\begin{aligned}
-2 \Delta a & <\bar{\nabla}_{i-1}(j)<2 \Delta a\left(\because \bar{\nabla}_{i-1}(j)=\nabla_{i-1}\right), \\
0 & <\bar{\nabla}_{i-1}(j)+2 \Delta a<4 \Delta a, \\
0 & <\bar{\nabla}_{i-1}(j-1)<4 \Delta a .
\end{aligned}
$$

Because $\bar{\nabla}_{i-1}(j-1)>0$, we take $M_{2}$ and move to $(i, j)$.

Case 2.2. Start from $(i-1, j):(i, j)$ can be reachable using $\bar{\nabla}_{i-1}(j)$ because $\bar{\nabla}_{i-1}(j)=\nabla_{i-1}$.

Now we assume that $(i, j) \in \mathscr{V}(\Delta a, \Delta b)$ is reachable from $(i-k, l) \in \mathscr{B}(i, j)$ for $k \geq 1$, i.e., the points $k$ step backward from it. Because in general we can move to $(i-k, l) \in \mathscr{B}(i, j)$ from the points $k+1$ step back from $(i, j)$ by taking either $M_{1}$ or $M_{2}$, we consider only two special boundary cases of $(\Delta a-\Delta b, 0)$ and $(\Delta b, \Delta b)^{6}$ to check whether the movement from those points still belongs to $\mathscr{B}(i, j)$.

Case 1. $(i-k-1, l)=(\Delta a-\Delta b, 0)$ : We take $M_{2}$ and the next point belongs to $\mathscr{B}(i, j)$ because $\bar{\nabla}_{\Delta a-\Delta b}(0)=2 \Delta b(\Delta a-\Delta b)>0$.

Case 2. $(i-k-1, l)=(\Delta b, \Delta b)$ : We take $M_{1}$ and the next point belongs to $\mathscr{B}(i, j)$ because $\bar{\nabla}_{\Delta b}(\Delta b)=2 \Delta b(\Delta b-\Delta a)<0$.

This completes the proof by mathematical induction.

\section{APPENDIX C \\ PROOF OF THEOREM 1}

Here we prove only Case 1 because Case 2 follows from Case 1 and is just its application.

Case 1. $\frac{D}{A}<1$ : If $(i, j) \in \mathscr{V}(\Delta a, \Delta b)$, we obtain the following from Lemma 1 and Definition 3 .

$$
\begin{aligned}
-2 \Delta a & <\bar{\nabla}_{i}(j)<2 \Delta a, \\
-2 \Delta a & <2(i \Delta b-j \Delta a)<2 \Delta a, \\
-2 A & <2(i D-j A)<2 A(\because \Delta a=A, \Delta b=D) .
\end{aligned}
$$

Hence (10). If $i \frac{D}{A}$ is not an integer, it is clear from 10 that there are two values satisfying the inequality. If we also include the effect of the precision loss, there could be even more values satisfying the extended inequality of (11).

Let $k, \ldots, k+l$ be those values satisfying (11). Because $(i-l, k)$ belongs to $\bigcap_{m=0}^{l} \mathscr{B}(i, k+m)$ as per $(8)$, we can reach from $(i-l, k)$ to a valid point-i.e., one of $(i, k), \ldots,(i, k+l)$ - by applying Bresenham's algorithm with $\bar{\nabla}_{i-l}(k)$ by Lemma 2 .

\section{REFERENCES}

[1] Y.-C. Wu, Q. Chaudhari, and E. Serpedin, "Clock synchronization of wireless sensor networks," IEEE Signal Process. Mag., vol. 28, no. 1, pp. 124-138, 2011.

[2] X. Huan and K. S. Kim, "On the practical implementation of propagation delay and clock skew compensated high-precision time synchronization schemes with resource-constrained sensor nodes in multi-hop wireless sensor networks," Computer Networks, vol. 166, pp. 1-8, Jan. 2020.

${ }^{6}$ See the points $(2,0)$ and $(4,4)$ in Fig. 2 as an example. 
[3] J. E. Bresenham, "Algorithm for computer control of a digital plotter," IBM Systems Journal, vol. 4, no. 1, pp. 25-30, 1965.

[4] R. T. Rajan and A.-J. van der Veen, "Joint ranging and clock synchronization for a wireless network," in Proc. CAMSAP 2011, Dec. 2011, pp. 297-300.

[5] J.-P. Sheu, W.-K. Hu, and J.-C. Lin, "Ratio-based time synchronization protocol in wireless sensor networks," Telecommunication Systems, vol. 39, no. 1, pp. 25-35, Sep. 2008.

[6] K. S. Kim, S. Lee, and E. G. Lim, "Energy-efficient time synchronization based on asynchronous source clock frequency recovery and reverse two-way message exchanges in wireless sensor networks," IEEE Trans. Commun., vol. 65, no. 1, pp. 347-359, Jan. 2017.
[7] B. Etzlinger, N. Palaoro, W. Haselmayr, B. Rudić, and A. Springer, "Timestamp free synchronization with sub-tick accuracy in the presence of discrete clocks," IEEE Trans. Wireless Commun., vol. 16, no. 2, pp. 771-783, Feb. 2017.

[8] K. S. Kim, "Asynchronous source clock frequency recovery through aperiodic packet streams," IEEE Commun. Lett., vol. 17, no. 7, pp. 14551458, Jul. 2013.

[9] Texas Instruments, "Selection and specification of crystals for Texas Instruments USB 2.0 devices," Application Report, Dec. 2002, accessed: 25 August 2021. [Online]. Available: https://www.ti.com/lit/an/slla122/ slla122.pdf

[10] TinyOS. [Online]. Available: https://github.com/tinyos/tinyos-main 